\title{
The Paradox of Analogy
}

\section{DAVID BotTING}

Institut de Filosofie da Linguagem

FCSH

Universidade Nova de Lisboa

LISBON, PORTUGAL

davidbotting33@yahoo.co.uk

\begin{abstract}
I will show that there is a type of analogical reasoning that instantiates a pattern of reasoning in confirmation theory that is considered at best paradoxical and at worst fatal to the entire syntactical approach to confirmation and explanation. However, I hope to elaborate conditions under which this is a sound (although not necessarily strong) method of reasoning. It does not, as its exponents claim, instantiate a pattern of reasoning distinct from deductive and inductive logic.
\end{abstract}

Résumé: Je vais montrer qu'il existe un type de raisonnement par analogie qui repress ente un modèle de raisonnement dans la théorie de confirmation qui est au meilleur paradoxal et, au pire, fatal à toute l'approche syntaxique sur la confirmation et l'explication. Cependant, j'espère élaborer les conditions dans lesquelles ce raisonnement est solide (mais pas nécessairement très fort). Il ne représente pas, comme l'affirment ses exposants, un modèle de raisonnement distincte de la logique déductive ou inductive.

Keywords: analogy, analog, confirmation, induction, analogical reasoning

\section{The Role Of The Universal Claim}

Much of the discussion around analogy revolves around the following decision tree:

$\lceil$ Are analogical arguments really arguments?

(NO)

(YES)

All analogies are 'figurative' Are they (D)eductive arguments, They have heuristic and perhaps rhetorical functions (I)nductive arguments, or some (Allen, Kaptein, Agassi)

(O)ther kind of argument?

$\begin{array}{cll}\text { I }^{\text {I }} & \text { (I) } & \text { (O) } \\ \text { Woods \& Hudak } & \text { Wreen } & \text { Govier } \\ \text { Agassi } & \text { Govier } & \text { Barker } \\ \text { Waller } & \text { Waller } & \text { Guarini }\end{array}$

(C) David Botting. Informal Logic, Vol. 32, No. 1 (2012), pp. 98-115. 
Note that these are not exclusive. Most philosophers concede that some analogical arguments are inductive and some are figurative. Govier, Guarini and Barker hold that there is another class of analogical arguments - called by Govier (1989) "a priori analogies" - that are arguments concerning how something is to be classified without being either deductive or inductive.

If such arguments are valid, then the tradition that separates all arguments and reasoning into deduction and induction is incomplete. Recently there has been a proliferation of such kinds of reasoning: abductive reasoning, the plausibilistic reasoning of Rescher and the closely related presumptive reasoning of Walton, and the conductive reasoning of Wellman, to name a few. ${ }^{1}$ Personally, I am not yet ready to do away with tradition. In this paper, I wish to uphold tradition with regard to analogical arguments. Those other forms of reasoning will be treated elsewhere.

There are broadly three strategies that might be taken to uphold tradition. We may hold that a priori analogies are not arguments at all. This strategy is taken on the left of the decision tree where all analogies, a priori or not, are only figurative. A more modest position is available (but not, as far as I can tell, occupied by anyone) that a priori analogies are figurative although other kinds of analogies are not. The second and third strategies attempt to provide an analysis that assimilates a priori analogies to well-recognized and understood forms of argument: the second to deductive arguments (see Waller 2001) and the third to inductive arguments (see Wreen 2007). I will be adopting this third strategy: I will be arguing, taking Hempel as my inspiration, that a priori analogies instantiate a pattern of reasoning familiar from confirmation theory, although there condemned as paradoxical. It is a kind of inductive reasoning that, although not strong generally speaking, is nonetheless valid.

\footnotetext{
1 I have argued elsewhere that many abductions are semantic entailments, and hence deductive in so far as they are valid in virtue of meaning. I have also written elsewhere that presumptive reasoning is simply deductive (although many philosophers prefer to call this "inductive" on the grounds that the major premise is an inductive generalization) reasoning where a probability has been attached to the consequence relation, as in Hempel's I-S model. Note that I do not generally object to these forms of reasoning as alternative models of reasoning. If presumptive reasoning were presented as only a model of reasoning and nothing more I would find far less to object to. The error is in drawing the conclusion that this is a conceptually distinct kind of reasoning from what, in the end, are only features of the model. I find Walton especially prone to this error.
} 


\subsection{The First Strategy}

All so-called arguments from analogy, say philosophers on the left of the decision tree, lead to deductive arguments whose universal claim subsumes both the new case in which you are trying to draw an inference (the 'target') and the analogous case in which the conclusion is already decided (the 'source'). The function of the analogon is not to license any inference but to suggest and refine this universal claim, and it is this claim that licenses the inference. Analogical arguments are not really arguments but are heuristics, a way of discovering deductive and perhaps inductive arguments.

Kaptein $(2005,502)$ puts it like this: "[T]he whole weight of so-called argument from analogy is on underlying principle(s) and not on the original analogon at all. Then the original analogon is no more than a suitable starting-point from a purely heuristic point of view, or a rhetoric prop...." Agassi (cited in Gamboa 2008, 232) puts it in the form of a dilemma: "For any putative argument by analogy, either (1) the thinking is merely suggestive (in the heuristic sense) and does not establish any conclusion, or (2) the argument can be analysed into a nonanalogical form." In (1) Agassi repeats Kaptein's view, but in (2) Agassi seems to concede that some arguments from analogy are genuinely arguments, and that the universal claim only hinted at in (1) is in some way already implicit in the contents of the analogy in (2) when it has been properly understood, which is to say, when the resemblance has been analysed such that the question of why the two cases are relevantly similar, or why one similarity is relevant whilst another is not, has been answered.

Here is an example from Kaptein (2005, 501-502):

Waking up in the morning, a man travelling on a river steam boat found money missing from his cabin. He sued the steamboat company for damages. Though there was no precedent, the court still ordered the company to pay, because innkeepers had such liability by precedent and because 'steamboat circumstances' were found to be sufficiently like 'inn circumstances', regarding the legal issue at hand.

What matters here is the underlying principle, and nothing else. If there is a general duty or obligation of care on parties offering night accommodation, then both inn-keepers and steamboat companies are under such a duty or obligation. But of course there is no logical relationship at all between the original analogon (the inn precedent) and the general principle of care. Things are the other way round: if the general principle holds, than there are inn liability implications, and steamboat com- 
pany implications as well. But the inn liability rule may also be derived from the (patently implausible) rule that innkeepers are liable for anything happening to their guests, and from many more patently implausible 'principles'.... If on the other hand no general principle of care as stated above is taken to hold, then a duty of care for innkeepers must be a special case, or an antiquated precedent.

Here Kaptein seems to reject the view that the rule or universal claim can be found, so to speak, simply by understanding the analogy, because there are indefinitely many rules that could, logically, subsume both cases; to think that the analogon somehow picks out a specific rule and argument amounts to affirming the consequent, to imagining a logical relation to exist where there is none. If we had $p \supset r$ and $q \supset r$ ( $p$ and $q$ being possible rules and $r$ being the analogon) then we could not infer $p$ from $r$ or $q$ from $r$. The rule is not to be revealed by any analysis but specified by a decision, and once specified it follows immediately that the new case falls under the rule and the verdict in the new case is determined by it without any help from the analogon.

Although Kaptein's logic is obviously impeccable I am not sure it is sufficient for the claim that analogies can only be figurative and cannot be deductive arguments. Granted, there may be an indefinitely large set of arguments that are logically compatible with the analogy, but it does not follow that, in giving the analogy, the arguer is giving this entire set or nothing from the set; one member of the set of logically compatible arguments is indeed a sound argument, and if it is plausible to reconstruct the argument that is given as this argument, it seems wrong to say that this is not the argument given by the arguer simply because some other argument, less plausible as a reconstruction, is logically possible. Kaptein's confessedly "silly" rules would not be plausible as reconstructions of the argument being offered; they are not believed, even dispositionally, by the arguer, and such a reconstruction would be rejected by the arguer as misrepresenting his argument or even as committing the fallacy of the straw man.

\subsection{The Second Strategy}

Some analogies function in the way Kaptein and Agassi (in the first horn of his dilemma), say; these are figurative analogies and are not arguments. Is Kaptein's example above a figurative analogy or an argument? It fits Govier's definition of an a priori analogy. Analogical arguments of this kind are concerned with 
how something should be classified, and are usually reconstructed in the following deductive form:

(1) $A$ has $x, y, z$

(2) $B$ has $x, y, z$

(3) $A$ is $W$

(4) All things that have $x, y, z$ are $W$ [universal claim]

(5) Therefore, $B$ is $W$

The universal claim is suppressed in the argumentation offered, and the issue is whether belief in, or perhaps commitment to, this claim can always be plausibly ascribed to the arguer. As Kaptein has shown, commitment to the universal claim is not logically implied by commitment to (1), (2) and (3). Is there some other way in which commitment to the universal claim can be ascribed to the arguer? Agassi seems to say that this arises out of understanding why the properties $x, y$, and $z$ make $A$ and $B$ analogous.

Waller (2001) endorses a similar view; accepting the distinctness of the class of a priori analogies he holds that all arguments in this class have this deductive form. Waller is here adopting the second strategy. Govier and Guarini claim it cannot, and if it cannot, then although analogies can be reconstructed as above, such is not a plausible reconstruction of what the arguer is really arguing and is irrelevant to whether the arguer is arguing well, i.e., is personally justified in taking her conclusion to follow from her premises. The arguer, Govier would say, takes her conclusion as warranted even in the absence of (4) - (1), (2), (3) therefore (5) being a valid argument despite not being deductively valid or inductively strong-and is not mistaken in drawing this conclusion.

Govier (1989, 141-44) notes about the deductive reconstruction that once the universal claim is included as an implicit premise then (2) and (4) entail (5) whilst (1) and (3) (the source or analogon) are redundant. In fact, it seems that Govier concedes that there are cases where the arguer can plausibly be ascribed the universal claim, in which cases this argument-form accurately reconstructs the argumentation offered. But there are other cases when it is not plausible, e.g., if the universal claim is difficult to formulate or more dubious than the conclusion of the argument, and although a universal claim is involved, it does not play the role of licensing an inference and is not really a premise at all but is only a background assumption (Govier 1989, 14647). We can make valid inferences, Govier says, without the contribution of a universal claim.

Waller (2001) objects that Govier has misconstrued the role of the universal claim. Granted that it may be difficult to 
formulate the universal claim, but, assuming that we are entitled to say that the two cases are relevantly similar at all, there must be some rule, however vague, that can be tentatively proposed as answering why such and such a similarity is relevant, which then evolves through a reflective process of mutual adjustment between the claim, the analogy, and possible dissimilarities and counter-analogies. We do not need to suppose, as Govier seems to, that the universal claim be better known than the conclusion; our knowledge of both increases together with neither the rule nor its instances having epistemic or logical priority.

Waller, and Govier (2002) in her response, each concede something to the other. Govier concedes that there may be mutual adjustment between the universal claim and the instance falling under it but notes that since this proceeds after the argument has been offered, it cannot be what the arguer meant; even if it can be reconstructed as a deductive argument this does not mean that it is plausible to reconstruct it as a deductive argument. $^{2}$ I think this is right, and when Waller $(2001,205)$ concedes that "in some cases we can indeed evaluate the analogical argument without ascertaining the underlying principle" he has conceded all that Govier needs. Guarini (2004, 157) concurs with this assessment and points out further that the respondent in the argumentation may well be diametrically opposed to the rule, and hence that offering the deductive argument where the rule has been formulated would be a waste of time and almost certainly fail whereas the analogical argument might succeed. There may even be cases where the arguer finds herself endorsing a rule that, prior to reflection on the analogy, she would have rejected. It is completely implausible to ascribe belief, even of a dispositional sort, in such a rule to the arguer; if anything, it is more plausible to ascribe belief in its negation. ${ }^{3}$

2 The fact that many arguments are logically compatible with the analogy does not mean that they are all equally plausible as reconstructions, and even if we are determined to reconstruct the analogy as a deductive argument, there is a whole range of deductive arguments that are potential reconstructions because consistent with the analogy, as shown in the discussion of Kaptein. The fact that the argument can be reconstructed deductively does not mean that this is how the argument should be reconstructed and does not even solve the problem of what the universal claim is, without which the mere statement that it has a deductive form is vacuous and unhelpful.

${ }^{3}$ I am not sure that Waller could not respond to this along the lines that the arguer is mistaken about what rule she endorses and that the analogy, in presenting her with a counter-instance to her rule, causes her to adjust her rule to accord with her judgment in the analogous case. One might think of Frankfurt-cases where, because of our intuitions about responsibility in these cases, we come to the conclusion that our concept of responsibility is not, perhaps, what we thought it was or would have said that is was if asked. We do not say that our intuitions start to track a different concept when we encounter 
I do, however, have a nagging worry that Govier's position is incoherent. Can an arguer really make relevance judgments without having some idea of a rule, however vague, underlying why it is those features and not others that are relevant? Note that the features named in the argument are not all the features of the cases, or even all the features that are common to both cases, but only those in virtue of which something is to be classified as $\mathrm{W}$, and once this is decided there seems little for the analogon to do. The argument-form offered by Govier eschews the universal claim but, because some properties are selected and some are not, it remains the case that there must be some general rule underlying this choice to which belief can be ascribed. This is a version of Agassi's point about understanding the analogy. Perhaps Govier would concede that the arguer does have some idea of a rule, albeit as a background assumption that governs her judgments of relevance without necessarily enabling her to spell them out. I think that the experience of feeling two things to be similar without being able to say exactly how they are similar is not too unfamiliar, but I question whether this is sufficient, given what has been conceded about mutual adjustment.

I think that Guarini's suggestion for the argument-form of a priori analogies is superior because the conclusion is simply that the source and the target are to be treated in the same way:

(1) $a$ has features $f_{1}, f_{2}, \ldots f_{\mathrm{n}}$

(2) $b$ has features $f_{1}, f_{2}, \ldots f_{\mathrm{n}}$

(3) Therefore, $a$ and $b$ should be treated or classified in the same way with respect to $f_{\mathrm{n}+1}$

Clearly, (1) and (2) do not entail (3), i.e., it is not deductively valid and can be weakened or defeated by relevant dissimilarities and even unmentioned similarities. When

(4) $a$ is $W$ in virtue of $f_{1}, f_{2}, \ldots f_{\mathrm{n}}$

(5) Therefore, $b$ is $W$

are added, (3) and (4) do deductively ${ }^{4}$ entail (5) and form a deductive argument. What is established by the analogon, and is

Frankfurt-cases, we say instead that the concept was inadequately formulated and revise its formulation. Even prior to encountering Frankfurt-cases it does not seem to me implausible to ascribe belief in this revised formulation. Our intuited conclusion is justified because of the universal claim so formulated and our intuition can still be accurately reconstructed as a deduction.

4 Guarini $(2004,162)$ says that "the third and fourth claims entail the fifth." It is not clear whether by this he means deductively entail but this is the way I take it because it seems to me that he wants to break the argument into a non- 
sometimes what we really want to know, is that the two cases should be treated the same way, i.e., (3). Often this is the only decision we need to make and providing a specific classificatory term such as $\mathrm{W}$ is more a convenience than anything else. In other words, we have two steps: establishing (3) from (1) and (2) and establishing (5) from (3) and (4). This second step does not always appear and is not always necessary (Guarini 2004, 161-62). Because of this, and because even if it were necessary it is besides a deductive argument, the claim that a priori analogies instantiate a form of reasoning that is neither deductive nor inductive depends on showing that (1), (2) therefore (3) is not a deductive argument (which has been shown) and that it is not an inductive argument (which remains to be shown).

Guarini does not show that (1), (2) therefore (3) is not an inductive argument. Like the non-demonstrative arguments he seems to favour, inductive arguments do not need to refer to a universal claim and do accommodate varying degrees of strength. Moreover, Guarini's argument-form looks very like a second-order induction. Consider the second-order induction described by Reichenbach (1938). There have been repeated experiments aimed at melting samples of carbon, all of which have failed. A first-order inductive generalization leads to the conclusion that carbon doesn't melt. However, carbon has properties by which it is classified as a metal, and a second-order inductive generalization over metals shows that they do melt if the temperature is high enough. This supports the conclusion that carbon will also melt if you get the temperature high enough. Substituting "melts" for $\mathrm{f}_{\mathrm{n}+1}$ and "being a metal" for $f_{1}, f_{2}, \ldots f_{\mathrm{n}}$ into Guarini's argument-form seems to give you Reichenbach's argument. If it hadn't been the case that other metals melted, then there would be no reason to think it was the case for carbon either: so all metals are to be treated the same way, as Guarini says. Thus, Guarini's argument-form does not support the claim that a priori analogies instantiate a distinct kind of reasoning; they are distinct from ordinary inductive arguments only in being second-order.

deductive inference of (3) from (1) and (2)-which his paper goes to some lengths to analyse - and a (not always necessary) deductive part which is largely ignored in the paper. Furthermore, I think that it is a deductive entailment on the basis that (3) is making a general claim about some property referred to indeterminately by $f_{\mathrm{n}+1}$. The strength of this general claim depends on how closely $f_{n+1}$ follows $f_{1}, f_{2}, \ldots f_{\mathrm{n}}$ and this is strong when $f_{n+1}$ is $W$ given $W$ is a function of $f_{1}, f_{2}, \ldots f_{\mathrm{n}}$ as stated in (4). In other word, the conclusion follows deductively by a universal instantiation of (3) with $\mathrm{W}$ in place of $f_{\mathrm{n}+1}$. Also, since (3) is established on the basis of sharing $f_{1}, f_{2}, \ldots f_{\mathrm{n}}$ it would be inconsistent to treat $a$ and $b$ differently with respect to $f_{n+1}$ since this would amount to treating them differently with respect to $f_{1}, f_{2}, \ldots f_{\mathrm{n}}$. 


\subsection{The Third Strategy}

Guarini's argument-form looks inductive, although he would probably deny this. This leads us into the third strategy. Wreen $(2007,221-22)^{5}$ denies that a priori analogies are a distinct class-all arguments from analogy alike have one of two argument-forms, both of which are inductive. I will discuss only the first:

I. $\quad a$ has characteristics $f_{1}, f_{2}, \ldots f_{\mathrm{n}}$

II. $a$ also has characteristic $W$

III. $a$ 's having characteristic $\mathrm{W}$ is caught up with its hav$\operatorname{ing} f_{1}, f_{2}, \ldots f_{\mathrm{n}}$

IV. $b$ has characteristics $f_{1}, f_{2}, \ldots f_{\mathrm{n}}$

V. $b$ also has characteristic $W$

VI. b's having characteristic W is caught up with its having $f_{1}, f_{2}, \ldots f_{\mathrm{n}}$

VII. $x$ has characteristics $f_{1}, f_{2}, \ldots f_{\mathrm{n}}$

VIII. Therefore, $x$ has characteristic $W$

This is an induction by enumeration that attempts to establish the inductive generalization $\forall x .\left(f_{1}(x) \wedge f_{2}(x) \wedge \ldots \wedge f_{\mathrm{n}}(x)\right) \supset$ $W(x)$. It is unlike most inductive generalizations, however. For instance, suppose that $f_{1}, f_{2}, \ldots f_{\mathrm{n}}$ are the properties of ravenhood and $W$ is being black. Then, $a$ is a raven and $a$ is black confirms that all ravens are black. But here, as in most inductive generalizations, $a$ 's being $W$ can be established independently (in this case, by observation) of $a$ 's having $f_{1}, f_{2}, \ldots f_{\mathrm{n}}$ and the conclusion is a prediction to the effect that the next thing with $f_{1}, f_{2}, \ldots$ $f_{\mathrm{n}}$ will also be $W$. Being black is not a classification. Consider the difference when $W$ is a classification; for instance, where $W$ is ravenhood itself there is no way of establishing ravenhood beyond establishing $f_{1}, f_{2}, \ldots f_{\mathrm{n}}$ and it does not seem possible that the next thing with $f_{1}, f_{2}, \ldots f_{\mathrm{n}}$ could fail to be W. Indeed, this seems to be the point of premise (III): there seems to be no advantage here in finding more and more instances. The general form of an instance, viz., $\exists x .\left(f_{1}(x) \wedge f_{2}(x) \wedge \ldots \wedge f_{\mathrm{n}}(x)\right) \wedge W(x)$, does not establish $\forall x .\left(f_{1}(x) \wedge f_{2}(x) \wedge \ldots \wedge f_{\mathrm{n}}(x)\right) \supset W(x)$ by incremental confirmation only but, because all instances are on a par with respect to a classification such as this, by the rule of

${ }^{5}$ Guarini's and Wreen's argument-forms have both been adapted to emphasize their similarities and differences from each other and from Govier's argument-form. 
universal generalization - the universal generalization is a logical consequence of the existential generalization. The converse does not hold since the universal statement does not entail any existential statement (i.e., any particular) since it could be vacuously true, and the target, it should be noted, is a particular case. This is important because the confirmation relation is closed under logical consequence; we cannot argue, as we might like to, that whatever confirms the universal statement also confirms the target.

Despite their oddness arguments whose conclusion is a classification are inductive arguments and not non-inductive arguments. Govier makes much of the fact that a priori analogies do not make predictions (though this is contested by Wreen) and that it seems to make no sense to say, for example, that something can instantiate all the ravenhood-conferring properties yet fail to be a raven, but her conclusion that they are not inductive arguments does not follow. They are admittedly odd inductive arguments, but they are inductive arguments all the same, and their strength depends on how well the inductive generalization is confirmed.

This existential statement, then, has two tasks: to confirm the universal statement (which it also entails when a classification is involved) and to provide evidence for the corresponding existential statement in the target, since it need not be assumed that it is the universal statement confirmed directly by the source, rather than some universal statement of wider scope, that subsumes both source and target. Argument by a priori analogy, I hope to show in the next section, amounts to this second task: arguing for a probabilistic inference from the general form of an instance of the source to the general form of an instance of the target, thus establishing what the universal claim on its own cannot, namely that it has instances. Confirmation may then be transmitted, via universal claims that need never be ascertained at all and properties whose relevance to the classification need never be ascertained, from the source to the target. In this case, the source does confirm the target, and without any need to understand the analogy.

\section{The Role Of The Analogon}

I will now give a brief description of confirmation in the framework of Hempel's covering law model. First of all, there are two covering law models: the deductive-nomological (D-N) model and the inductive-statistical (I-S) model. I will distinguish these by using different conditionals, viz. 


$$
\left.\forall x .\left(f_{1}(x) \wedge f_{2}(x) \wedge \ldots \wedge f_{\mathrm{n}}(x)\right) \supset W(x)\right)
$$

is a categorical (universal material) conditional such that whenever the antecedent is satisfied, i.e., an $\mathrm{x}$ possesses the properties $f_{1}, f_{2}, \ldots f_{\mathrm{n}}$ then $x$ has $W$, that is to say, $f_{1}, f_{2}, \ldots f_{\mathrm{n}}$ are jointly sufficient conditions of $W$. This type of conditional is appropriate when $W$ is a classification, but it is by no means restricted to such cases since "All ravens are black" is normally treated as a categorical conditional yet nothing in the definition of blackness mentions any member of $f_{1}, f_{2}, \ldots f_{\mathrm{n}}$. The D-N model uses categorical conditionals. In contrast, the I-S model uses probabilistic conditionals $-f_{1}, f_{2}, \ldots f_{\mathrm{n}}$ not being sufficient conditions for $W$ but making it likely — which can be expressed as

$$
\left.\forall x .\left(f_{1}(x) \wedge f_{2}(x) \wedge \ldots \wedge f_{\mathrm{n}}(x)\right) \supset^{0.8} W(x)\right) .
$$

This is, on the relative frequency theory of probability, a singular (using a universal quantifier here is misleading in this respect) statement about the limit value of an infinite series of ratios dividing the frequency of instances of the consequent in the class of instances of the antecedent; in short, 8 out of 10 ravens are black. This makes sense, even if we have every reason to believe that all ravens are black. But it does not even make sense to say 8 out of 10 ravens possess the property of ravenhood; probabilistic conditionals are inappropriate for classifications and a priori analogies but may be appropriate for some inductive analogical arguments.

The role of the analogon in an a priori analogy is as a confirming instance in a D-N model. As already alluded to, the confirmation relation has certain properties in the D-N model. It is closed under logical consequence: if a hypothesis is confirmed, then any proposition entailed by the hypothesis is also confirmed. For instance, Raven(a) ^ Black(a) confirms the hypothesis $\forall x$. Raven $(x) \supset \operatorname{Black}(x)$, and since all ravens less than one year old are ravens, i.e.,

$$
\begin{aligned}
& \forall x \cdot \operatorname{Raven}(x) \supset \operatorname{Black}(x)) \vdash(\forall x . \text { RavenLessThanOneY- } \\
& \operatorname{earOld}(x) \supset \operatorname{Black}(x))
\end{aligned}
$$

then Raven $(a) \wedge \operatorname{Black}(a)$ confirms $\forall x$. RavenLessThanOneY$\operatorname{earOld}(x) \supset \operatorname{Black}(x)$ as well. This is the special consequence condition. Plausibly, confirmation is also closed under abduction/affirming the consequent, e.g., RavenLessThanOneYear$\operatorname{Old}(a) \wedge \operatorname{Black}(a)$ confirms, albeit to a lesser extent than it confirms its own universal generalization, $\forall x$. $\operatorname{Raven}(x) \supset \operatorname{Black}(x)$. 
This is the converse consequence condition. ${ }^{6}$ This condition was rejected by Hempel for reasons that are well-known and will be discussed later. For the moment, I just want to show how these conditions can be applied to a priori analogies.

The arguer believes that in both the source case and target case properties sufficient for establishing the classification (I will call these $W$-making) are co-instantiated. Let us assume for the moment that the properties are necessary as well as sufficient, i.e., let both cases instantiate precisely the same $W$-making properties. The problem with all the argument-forms discussed so far was that the properties mentioned in the actual argument had already been judged as $W$-making. Here that is not the case. Of course, in order to be in a position to use the analogy the arguer must believe that both cases are logically related, i.e., their universal generalizations are in the closure of the confirming instance. More than this, she does not strictly need logically speaking. It is a matter for empirical psychology to determine whether she does have some idea how they are so related.

Now drop the simplifying assumption of the last paragraph. When we are dealing with sufficient and not necessary properties it is entirely possible that the $W$-making properties in the source and target are completely disjoint, i.e., having no common member. Such a case would be a false analogy and the fact that a certain inference was licensed in the source would not mean that it was licensed in the target, even if, as a matter of fact, the target's premises and conclusion are true in the same models. This is an extreme case, though, and normally there would be an overlap, and the strength of the analogical inference depends on the amount of overlap. I think we can capture this in the following way. Symbolize the $\mathrm{W}$-making properties in the target as $W$-making $T$ and the $W$-making properties in the source

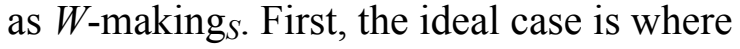

$$
\exists x . W \text {-making }(x) \supset \exists y . \mathrm{W}-\operatorname{making}_{T}(y)
$$

which is trivially the case when (although not equivalent to)

$$
\forall x .\left(W-\operatorname{making}_{S}(x) \supset W \text {-making }_{T}(x)\right)
$$

\footnotetext{
${ }^{6}$ Anything logically equivalent to an expression, e.g., its contrapositive, both entails and is entailed by it. What is often called the equivalence condition is thus just a special case of either of the conditions. The infamous paradox of the ravens illustrates how odd it is for a confirming instance to confirm both its universal generalization and the contrapositive of its universal generalization. See footnote 8 for a very brief discussion of this paradox.
} 
or, even more trivially, when $W$-making $_{T}$ is the same as or is materially equivalent to $W$-makings.

Generally, though, $\exists x . W$-making $s(x)$ will not entail $\exists x . W$ $\operatorname{making}_{T}(x)$ but only make it more probable by showing that at

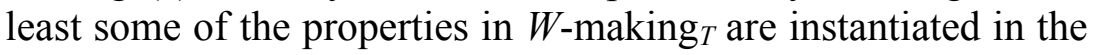
world and not, for example, ruled out by a physical law. This can be expressed by the inequality

$p\left(\exists x . W-\operatorname{making}_{T}(x) \mid \exists x . W\right.$-making $\left.(x)\right)>p(\exists x . W$ $\left.\operatorname{making}_{T}(x)\right)$.

Knowing that $\exists x . W$-making s $_{S}(x)$, itself a logical consequence by existential instantiation of $W$-making $(a)$, makes $\exists x . W$ $\operatorname{making}_{T}(x)$ more likely. ${ }^{7}$

There are, of course, well-known objections to using the special consequence condition and converse consequence condition together, and this is because by this means any true statement confirms any other true statement. For instance, "All ravens are black" is entailed by the hypothesis "All ravens are black and all men are mortal" and hence the latter is confirmed via the converse consequence condition whenever "All ravens are black" is confirmed, i.e., when a black raven is observed. "All men are mortal" is likewise entailed by the same hypothesis "All ravens are black and all men are mortal" which is confirmed via the converse consequence condition whenever "All men are mortal" is confirmed, i.e., when a mortal man is observed. However, since the hypothesis is confirmed and, via the special consequence condition, everything it entails is confirmed, it follows that "Here is a black raven" confirms "All men are mortal" and "Here is a mortal man" confirms "All ravens are black." This is at the least paradoxical and a good reason-Hempel and his followers have thought-to reject the converse consequence condition.

I think that this paradox can be dissolved if we use the inequality given above as a further constraint on the confirmation relation rather than simply a condition on good analogy. The question we have to ask ourselves in the example above is: does observation of a black raven make it more likely that there are men (disregarding the fact that presumably it is a man making the observation)? ${ }^{8}$ Perhaps it does, very weakly, since it shows

${ }^{7}$ Of course, it is a premise of the argument that the target has W-making properties and so our subjective probability of $\exists x . W$-making $_{S}(x)$ is 1.0 , but here we have to consider what would be the case if it were not a premise.

${ }^{8}$ A similar question seems to dispose of the paradox of the ravens. It seems odd, the paradox goes, that a black raven should confirm that all non-black things are non-ravens, or that a non-black non-raven, e.g., a white tie, con- 
that conditions necessary for life obtain, but this is something we knew anyway and was necessary for the asking of the question so, practically speaking, there is no confirmation here.

The target and not just its universal generalization, we can now say, is in the closure of the source under this modified confirmation relation; particulars can confirm particulars even without the help of auxiliary statements. The strength of the analogy depends on the value of the formula $p\left(\exists x . W\right.$-making man $_{T}(x) \mid \exists x . W$ $\left.\operatorname{making}_{S}(x)\right) / p(\exists x . W$-making $T(x))$. Applying this to Kaptein's example we have to ask whether the circumstances of the precedent make it more likely that there is exemplification of "steamboat circumstances." It seems to me that this is true. I can tell a story about how inns are resting places for travellers on long road journeys and cabins are resting places for travellers on long journeys by river, but it doesn't matter too much how I tell this story or reach this conclusion, and we can imagine it being reached in different ways; it is an empirical psychological question, and not a matter of being logically necessary for a good analogy, whether and in how many cases a universal claim (of some degree of vagueness) is entertained. The important point is that having reached this conclusion, precedents set in inn circumstances can be applied analogically to steamboat circumstances. The precedent is a confirming instance of the hypothesis "Everywhere inn circumstances obtain should be classified as $W$ " which confirms (for example) "Everywhere inn circumstances and steamboat circumstances obtain should be classified as $W$ " which confirms "Everywhere steamboat circumstances obtain should be classified as $W$ " which confirms "This steamboat circumstance should be classified as $W$."

\section{The role of the conclusion}

In a priori analogies the conclusion is a decision to classify something a certain way. In an inductive analogy the conclusion is typically (but, Wreen (2007) argues, is not always) a prediction, e.g., that the next raven to be observed will be black. This difference is relevant to the strength of the analogy because in the former case a single instance suffices to establish the generalization; it is logically impossible that there be a coinstantiation of raven-making properties in something that is not

firms that all ravens are black. Yet logically speaking, "All ravens are black" and "All non-black things are non-ravens" are equivalent. But the existence of a black raven gives no reason to believe that there are non-black things, and the existence of a white tie gives no reason to believe that there are ravens, so on the modified confirmation relation positive instances do not confirm the contrapositives of their universal generalizations. 
a raven. In many cases, I think that this is the only difference, because the generalizations in both cases are both expressed in the form of categorical conditionals: a priori analogies and inductive analogies involving only categorical conditionals have the same form.

Suppose that only $90 \%$ of observed ravens have been black, i.e., $\forall x$. Raven $(x) \supset^{0.9} \operatorname{Black}(x)$. This confuses the issue in two ways, which leads me to suggest that such arguments from analogy have a different form. Firstly, a probability statement cannot be verified or falsified by any singular statement, though it is perhaps still intelligible to say that a black raven confirms and a non-black raven disconfirms (but does not falsify) this statement. Secondly, it should be noted that $\forall x$. Raven $(x) \supset^{0.9}$ $\operatorname{Black}(x)$ does not have as a logical consequence $\forall x$. RavenLessThanOneYearOld $(x) \supset^{0.9} \operatorname{Black}(x)$. The special and converse consequence conditions do not seem to apply in the I-S model; it is consequently difficult to say what is in the closure of the confirming instance, if, indeed, anything is.

The argumentation in this kind of inductive analogy is, then, different not simply in terms of its strength but also in how it intends to license its conclusion. Its being a premise that the source's properties are sufficient for its conclusion, if the source thereby identifies that set of properties whose instantiation in the target is sufficient for the target's conclusion then this amounts to saying that all the relevant information has been considered (the condition of maximal specificity is satisfied) in the target argument and that its conclusion can be asserted absolutely and not just relative to the grounds of the argument. However, maximal specificity is a difficult condition to satisfy and we have no reason to suppose that it is satisfied in this case either.

There is, though, another way in which we might get the same result, which is when despite the reasons for and against the conclusion in the argument not containing all the possibly relevant information, they are nevertheless a representative sample of all possibly relevant information. That any proper subset of the relevant information gives the same probability to the conclusion as the whole set would be a massive mathematical fluke. But we may say that the degree to which the Wmaking properties in the source are a representative sample of those in the target is the degree of strength of the analogy. The fallacies of weak and false analogies become, on this view, biases in sampling. Maybe the fact that being a sample, unlike similarity (relevant or not), is not a symmetrical relation explains why analogies are normally only used in one direction. This kind of inductive analogy does seem to require some judgment about what is relevant. It can be considered as an enthy- 
meme where the statement about the representativeness of the sample is an unexpressed premise.

\section{The Role Of Understanding}

My concern in this paper has been to attenuate the role of understanding in the use of analogy. Agassi may be right that in understanding an analogy we must have some thoughts about what is and what is not relevant, but wrong in thinking that we can only infer on the basis of analogy if we understand the analogy and how the two cases are analogous; after all, there are many tools that we can use reliably and justifiably without knowing how they work, and analogy is no different. These judgments of relevance are generally formulated in the form of a universal claim, but Govier points out, correctly I think, that the arguer is not always basing her conclusion on such a universal claim, which is to say that she is not offering a deductive argument in which the universal claim is an implicit premise and she should not be reconstructed as doing so.

Inductive analogies do not involve universal claims, and some analogies are inductive analogies. However, according to Govier a priori analogies are not inductive either. Having discounted the possibility of its being a deductive argument, she concludes that this kind of analogy is a third kind of argument that is neither deductive nor inductive. I claim that they are inductive arguments in the genuine sense that they include a move from the particular to the general - the confirmation by singular statements of universal generalizations and, through the special consequence condition and an additional probabilistic claim, its substitution-instances. Certainly, this is a peculiar kind of inductive argument because, as I showed above, the consequent of the conditional is not observable independently of the antecedent, for which reason we may hesitate before calling it an empirical generalization, but this is a different matter from whether it is inductive, which in my view is a logical and not an epistemological question. Barker, Govier and Guarini confuse being em-

pirical with being inductive and reach the hasty conclusion that a priori analogies are not inductive arguments; even if the confirmation relation is redundant in the sense that a single instance suffices to establish a categorical conditional by universal generalization, this does not mean to say that the confirmation relation ceases to obtain, and it becomes relevant again when we consider a hierarchy of universal claims because confirmation, unlike certainty, can be transferred from the consequent to the antecedent. 


\section{David Botting}

However, even inductive arguments select which properties, out of all those available, are generalized over. This means that making this argument does require some judgments of relevance and this in turn means that the argument is plausibly reconstructed as a deductive argument, as Waller (2001) says. My account aims to substitute for a judgment of relevance a judgment about the relation between two existential statements, namely, the existential generalizations of the source and the target, and its success will depend on whether it is more plausible to ascribe (dispositional) belief in this probabilistic claim than it was in the universal claim. Making this judgment may, but also may not, involve making judgments of relevance. When these existential statements are strongly related then the analogy is strong because the source strongly confirms the target. An analogy is, I think, false if there is no relation at all, although Wreen (2007) offers a plausible argument that if one case is analogous to a second on the basis of sharing a property, and a second to a third on the basis of sharing a different property, then the first is analogous to the third despite that fact that it is entirely possible that there is no property that they both have in common. ${ }^{9}$ I think that this argument must be invalid because it seems to lead to the conclusion that everything is analogous to everything, but I confess that I have no refutation of it. It could be that the argument is valid but the conclusion is blocked by a judgment of irrelevance, just as some deductive arguments, though valid, are considered bad because the premises are irrelevant to the conclusion.

It does not seem possible to attenuate the role of understanding to the same extent in kinds of inductive analogy that do not contain classifications or properties not held to obtain universally. This is because we are here dealing with the I-S model

9 This is the second of Wreen's (2007) argument-forms:

I. $a$ has characteristics $f_{1}, f_{2}, \ldots f_{\mathrm{n}}$

II. $a$ also has characteristic $W$

III. $a$ 's having characteristic $W$ is caught up with its having $f_{1}, f_{2}, \ldots f_{\mathrm{n}}$

IV. $b$ has characteristics $f_{1}, f_{2}, \ldots f_{\mathrm{n}}, \ldots f_{\mathrm{n}+\mathrm{m}}$

V. $b$ also has characteristic $W$

VI. $b$ 's having characteristic $W$ is caught up with its having $f_{1}, f_{2}, \ldots f_{\mathrm{n}}$,

$$
\ldots f_{\mathrm{n}+\mathrm{m}}
$$

VII.

VIII.

IX.

X. $x$ has characteristics $f_{\mathrm{n}+\mathrm{m}}, f_{\mathrm{n}+\mathrm{m}+1}, \ldots f_{\mathrm{n}+\mathrm{m}+1}$

XI. Therefore, $x$ has characteristic $W$

Here $a$ has only one property in common with $b, b$ with $c$, and so on. Although he admits that the strength of this inference is weak, Wreen holds that it does give some reason to believe the conclusion. I think that this must be wrong, implying that "is analogous to" cannot be transitive, but I do not pretend to have refuted Wreen's argument. 
and the confirmation cannot traverse up and down the hierarchy of universal claims to its heart's desire - there is no unproblematic answer to the question of what is in the closure of the confirmation relation in the I-S model unless the condition of maximal specificity is satisfied, when it can be calculated by the probability calculus. In this case an analogy is strong if the reasons through which the conclusion of the source follows from its premises are representative of the reasons through which the conclusion of the target follows from its premises. An analogy is false if the sets of relations are disjoint. In offering this argument it seems to me that a judgment about relevance is necessary, and perhaps this argument could be given a reconstruction where the conclusion is taken to follow from the premises with a certain probability. Since this reconstruction would go from the universal to the particular and does not use confirmation I would, unlike the philosophers discussed here, call this a deductive argument despite the facts that probabilistic conditionals are involved and the conclusion cannot be detached.

There are two argument-forms for argument from analogy, but the inductive/a priori distinction does not align with the difference in form, which is rather due to the difference between the D-N model and the I-S model. A priori analogies and some inductive analogies use the D-N model and are the same in terms of form, although a priori analogies are, by definition, stronger, and so there is a point to making the distinction. Other arguments from analogy are statistical arguments.

\section{References}

Gamboa, Steven. (2008). "In defence of analogical reasoning." Informal Logic, 28(3): 229-241.

Govier, Trudy. (1989). "Analogies and missing premises." Informal Logic, 11(3): 141-152.

Govier, Trudy. (2002). "Should a priori analogies be regarded as deductive arguments?" Informal Logic, 22(2):155-157.

Guarini, Marcello. (2004). "A defense of non-deductive reconstructions of analogical arguments." Informal Logic, 24(2): 153-168.

Kaptein, Henrik. (2005). "Legal progress beyond pragmadialectics? Prospects beyond analogy and e contrario." Argumentation, 19: 497-507.

Reichenbach, Hans. (1938). Experience and Prediction. Phoenix Books. Chicago: The University of Chicago Press.

Waller, Bruce. (2001). "Classifying and analyzing analogies." Informal Logic, 21(3): 199-218.

Wreen, Michael. 2007. "A second form of argument from analogy." Theoria $73(3)$ : 221-239. 